\title{
Impact of the Surgical Approach: A Comparative Study between Transcanal and Posterior Tympanotomy Approach for Cochlear Implantation
}

\author{
Neeraj Suri ${ }^{1}$ Satya Sandilya ${ }^{1} \quad$ Ruta Sayani ${ }^{1} \quad$ Amit Anand ${ }^{2}$ \\ 1Department of ENT, GMERS Medical College, Gandhinagar, Gujarat, \\ India \\ 2Department of Audiology, Asian Speech and Hearing Clinic, \\ Ahmedabad, Gujarat, India \\ Address for correspondence Neeraj Suri, MS, Department of \\ ENT, GMERS Medical College, A/94, Shantam Towers, Behind Civil \\ Hospital, Near Amul Garden, Shahibaug, Ahmedabad 380004, \\ Gujarat, India (e-mail: drneerajsuri@outlook.com).
}

Ann Otol Neurotol:2020;3:10-15

\begin{abstract}
Introduction The aim of our study is to compare the surgical complications of the transcanal and posterior tympanotomy approach and to evaluate the advantages and disadvantages of both techniques.

Materials and Methods It is a prospective study involving 252 pediatric cochlear implant patients operated in the Department of ENT, GMERS Medical College and General Hospital, Gandhinagar, Gujarat, India. Out of these, 126 patients were operated by transcanal approach (group A) and 126 patients were operated by posterior tympanotomy approach (group B).

Results No significant difference in the mean duration of surgery $(p>0.064)$ was observed in both the groups. Major complications occurred in $60.2 \%$ of group $A$ and $3.1 \%$ of group B and minor complications occurred in $65 \%$ of group $A$ and $3.1 \%$ in group B, which is highly significant $(p<0.0134)$. The categories of auditory perception, speech intelligibility rating scales, meaningful auditory integration scale, and meaningful use of speech scale were assessed in both groups.

Keywords Conclusion Complication rate in the transcanal approach is higher as compared

- transcanal

- posterior tympanotomy

- cochlear implant with posterior tympanotomy approach. A complete alignment and introduction of electrode array into the basal turn of cochlea is more favorable in the posterior tympanotomy approach. Transcanal technique even as an alternative may not be useful. Outcomes may be affected depending upon the technique chosen.
\end{abstract}

\section{Introduction}

Cochlear implant (CI) surgery is an effective and doable alternative to restore hearing in cases of bilateral severe to profound hearing loss in patients who do not benefit from using an individual sound amplification device. ${ }^{1} \mathrm{CI}$ surgery is now being performed for more than 40 years. Different surgical approaches have been adopted for cochlear implantation, with cortical mastoidectomy and posterior tympanotomy being the most commonly followed techniques., ${ }^{2,3}$ So far, the reports of facial nerve injury in posterior tympanotomy approach have been reported as less than $1 \%$ with some studies reporting 1.7 to $2 .{ }^{3}$ Surgical expertise is required for the posterior tympanotomy approach and also the surgeon's uneasiness while drilling near the facial nerve has led to the development of alternative approaches with surgeons taking up the transcanal approach as the technique of choice in Asian setups. ${ }^{4}$ Non-mastoidectomy approaches include transcanal approach and other modifications like suprameatal and pericanal approaches for electrode insertion. ${ }^{4-7}$ The transcanal approach is where an active electrode is inserted through a tunnel created in the posterior superior bony meatal wall and cochleostomy. Some authors find these techniques crucial and helpful in cases of anatomical constraints 
such as small mastoid cavities, narrow facial recess, unusual course of sigmoid sinus, high and dehiscent jugular bulb, low lying dura, cochlear anomalies, where the posterior tympanotomy approach may be a surgical challenge. ${ }^{7.8}$ In lieu of surgeons now doing transcanal approach as the technique of choice in cochlear implantation, we did a comparative study. This study discusses the rate of complications between the transcanal approach and posterior tympanotomy approach in cochlear implantation and reviews the pros and cons of both approaches.

\section{Materials and Methods}

This is a prospective study, conducted on pediatric CI patients, who were operated at the department of ENT, GMERS Medical College and Civil Hospital, Gandhinagar, Gujarat, India from January 2015 to June 2016. The study included 252 patients. Of these, 126 cases were operated by transcanal approach which is group A and 126 cases by posterior tympanotomy approach group B. Cases with inner ear anomalies were excluded.

Ethical considerations: the study was approved by the Central Research Committee and Institutional Ethics Committee after thorough considerations.

\section{Preoperative Evaluation}

All patients were subjected to a routine ENT examination and audiological evaluation which included audiometry, brainstem-evoked response audiometry, auditory steady state response, otoacoustic emission, and tympanometry to assess the degree and type of hearing loss and the status of the middle ear. This was followed by radiological evaluation with high resolution computed tomography temporal bone and magnetic resonance imaging brain with membranous labyrinth to study the status of bony and membranous labyrinth to rule out any inner ear anomalies, facial and vestibulocochlear nerve anomalies. All patients underwent psychological, pediatric, cardiac, and routine hematological evaluation. All patients planned for surgery were given pneumococcal and meningococcal vaccination at least 1 month prior to surgery. Both groups underwent intraoperative neural response telemetry and impedance. Intraoperative " $C$ " arm imaging in transorbital view/Stenvers views was done to confirm proper functioning of the device and correct placement before extubation so that any malpositioning or kinking can be corrected immediately.

\section{Postoperative Period}

All patients were given antibiotics for a period of 1-week postoperatively. Mastoid dressing was opened after 4 days. The surgical complications were classified into major and minor according to the criteria described by Cohen and Hoffman.

\section{Postoperative Evaluation}

The auditory receptive abilities and speech intelligibility of the patients were rated using the categories of auditory perception (CAP) and speech intelligibility rating scales (SIR), respectively. Other than these two rating scales, the meaningful auditory integration scale (MAIS) and meaningful use of speech scale (MUSS) were used to monitor the development of auditory stages and oral language. All the patients underwent regular auditory verbal training as well as speech and language therapy.

\section{Results}

\section{Baseline Characteristics}

A total of 252 patients underwent cochlear implantation surgery for bilateral profound sensorineural hearing loss between January 2015 to June 2016. All patients belonged to the pediatric age group ranging between 8 months to 4 years. In group $A$, the electrode array was inserted through the cochleostomy, i.e., 34 patients (26.98\%) and in group B, the electrode array was inserted through the round window in 92 patients (73\%). All patients were followed up to 4 years post-surgery.

\section{Minor Complications}

Minor complication occurred in 65\% of group A cases and $3.1 \%$ of group B cases which required prolongation of hospital stay. Complications related to skin flap (necrosis, edema, delayed wound healing) were in $2.38 \%$ of cases of group A and $0.793 \%$ of group B. Patients responded well to antibiotic therapy and repeated outpatient wound dressing ( - Table $\mathbf{1}$ ). Neurological complications manifesting as facial nerve palsy were observed in the immediate postoperative period in $4.76 \%$ of group A cases and $1.58 \%$ of group B cases. In group B, it is related to the damage by excessive heat dissipated by drilling or anteriorly lying facial nerve, whereas in group A we attribute it to its blind drilling of the tunnel. Chorda tympani injury was due to mobilization of tympanomeatal flap and drilling of the tunnel in $8.7 \%$ of group A cases. External ear canal granuloma was seen only in group $A$ due to end aural incision and elevation of tympanomeatal flap. It was treated under microscopic examination and application of trichloroacetic acid. Posterior meatal injury was seen intraoperatively in group A due to angulated ear canal and for tunnel drilling ( - Fig. 1).

Table 1 Minor complications of cochlear implant surgery in both groups

\begin{tabular}{|l|l|l|}
\hline $\begin{array}{l}\text { Minor } \\
\text { complications }\end{array}$ & $\begin{array}{l}\text { Transcanal } \\
\text { approach } \\
\text { (group A, } \\
\boldsymbol{n}=126)\end{array}$ & $\begin{array}{l}\text { Posterior } \\
\text { tympanotomy } \\
\text { approach } \\
\text { (group B, } \boldsymbol{n}=126)\end{array}$ \\
\hline $\begin{array}{l}\text { Transient peripheral } \\
\text { facial palsy }\end{array}$ & $6(4.76 \%)$ & $2(1.58 \%)$ \\
\hline $\begin{array}{l}\text { Posterior meatal wall } \\
\text { injury }\end{array}$ & $51(40.4 \%)$ & 0 \\
\hline $\begin{array}{l}\text { Chorda tympani } \\
\text { nerve injury }\end{array}$ & $11(8.73 \%)$ & $1(0.793 \%)$ \\
\hline Skin flap infections & $3(2.38 \%)$ & $1(0.793 \%)$ \\
\hline EAC Granulation & $11(8.73 \%)$ & 0 \\
\hline
\end{tabular}

Abbreviation: EAC, external auditory canal. 


\section{Major Complications}

Sixty percent in group A cases and 3.17\% in group B cases had complications mainly related to electrode insertion and surgical revision. Surgical revision was required in seven cases

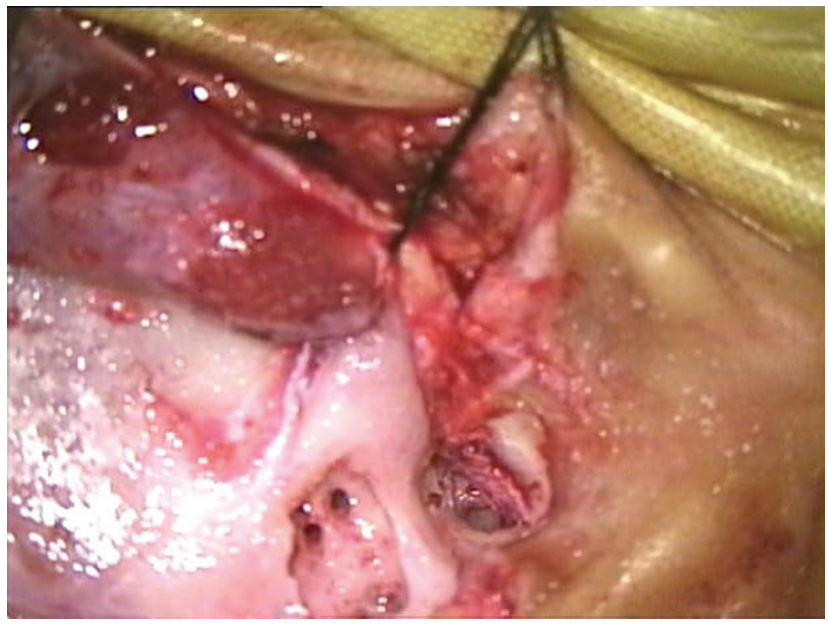

Fig. 1 Breaking of the tunnel while drilling in group A.

Table 2 Major complications of cochlear implant surgery in both groups

\begin{tabular}{|l|l|l|}
\hline Major complications & $\begin{array}{l}\text { Transcanal- } \\
\text { approach } \\
\text { (group A, } \\
\boldsymbol{n}=126)\end{array}$ & $\begin{array}{l}\text { Posterior } \\
\text { tympanotomy } \\
\text { (group B, } \\
\boldsymbol{n}=26)\end{array}$ \\
\hline $\begin{array}{l}\text { Electrode insertion } \\
\text { problems }\end{array}$ & $37(29.36 \%)$ & $2(1.58 \%)$ \\
\hline $\begin{array}{l}\text { Tympanic membrane/ } \\
\text { angle injury }\end{array}$ & $15(11.9 \%)$ & 0 \\
\hline $\begin{array}{l}\text { Flap dehiscence/ } \\
\text { infection }\end{array}$ & $5(3.96 \%)$ & $1(0.793 \%)$ \\
\hline $\begin{array}{l}\text { TM retraction/ } \\
\text { cholesteatoma }\end{array}$ & $2(1.58 \%)$ & $1(0.793 \%)$ \\
\hline Ear discharge & $17(13.49 \%)$ & 0 \\
\hline
\end{tabular}

Abbreviation: TM, tympanic membrane.

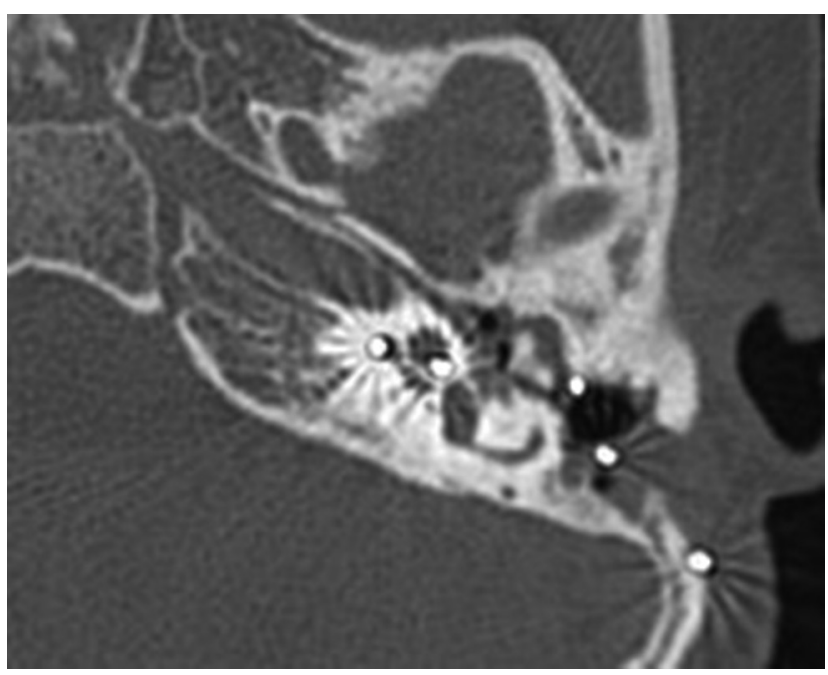

Fig. 2 Disease in middle ear and attic with implant in situ in group A. in group A. They had residual post otitis perforated eardrum. Two cases had thinned tympanic membrane due to retraction pocket through which the electrode was seen $(2$ years after the first implantation). Myringoplasty was done in all (-Table 2). One case in group A had cholesteatoma (-Fig. 2). Explantation was done in two cases in group A probably due to local skin flap responsible for wound dehiscence and the rest were managed by prolonged bandage and dressing. One case of group B was managed by pedicled temporal muscle reconstruction. 29.3\% in group A had issue in electrode insertion probably due to basal turn of cochlea positioned at more posterior angle than the ear canal (-Figs. 3-5). Local skin complications were statistically significant in group A with $p<0.0546$. The minor and major complications were significantly higher in group A with $p$-value $<0.013$.

\section{Outcomes}

All the patients were grouped into two with respect to age for assessing the audit and speech-language development (-Table 3). Mean scores were divided into two groups with respect to age ( - Table 4$)$.

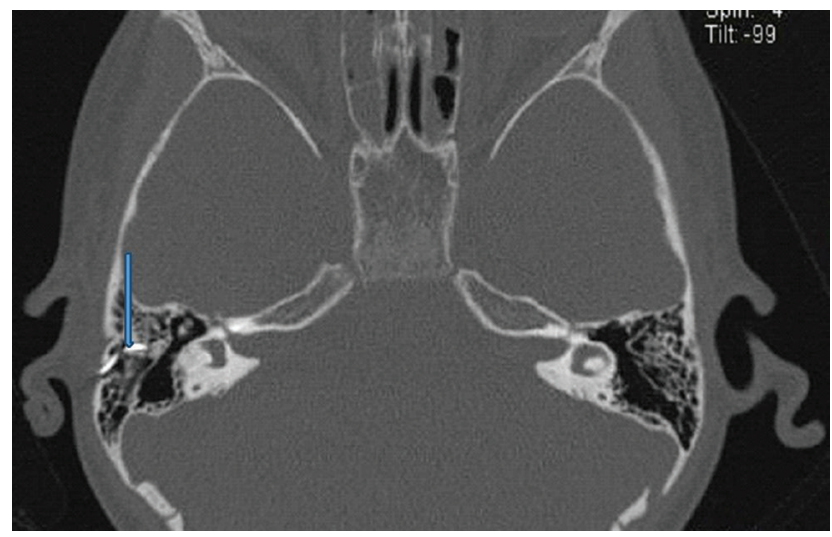

Fig. 3 The electrode at the entry point of tunnel (blue arrow) pointing at the first bent.

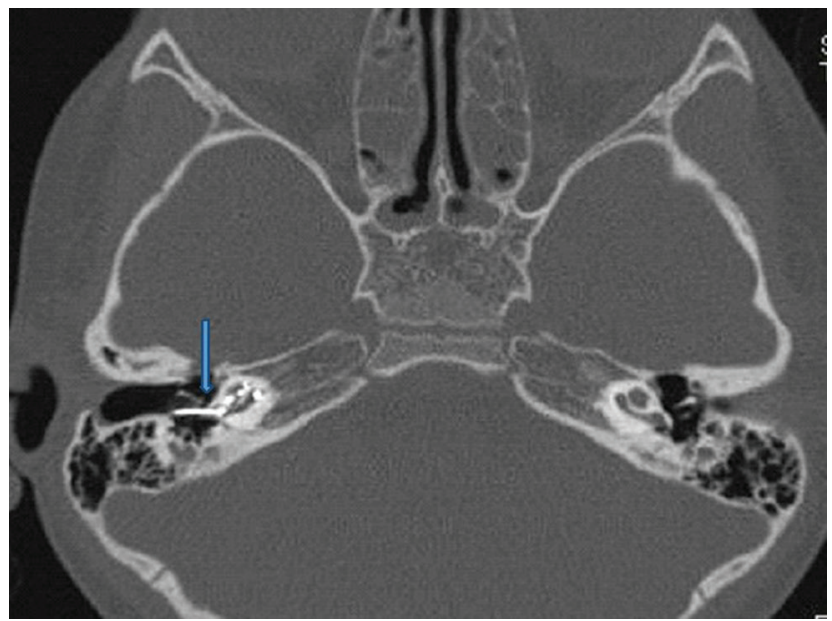

Fig. 4 Entry of the electrode to the basal turn of the cochlea is at a significant angle to the axis of the segment of the basal turn. 
All the tests scores were compared and analyzed between these two groups. CAP, SIR, MAIS, and MUSS scores in both groups were increasing with time by regular use of the device. Age group 0 to 1 year had a significant difference in all the tests and $p$-value is $<0.05$. Group B patients had better performance than group A. Age group 1.1 to 2 years had similar test results which showed no significant difference in CAP and SIR as they performed equally in both the groups with the $p$-value of $>0.05$. They had significant differences in MAIS and MUSS with the $p$-value of $<0.05$. Age group 2.1 to 3 years had greater difference in the scores across the tests except for MAIS. We found significant differences in CAP, SIR, and MUSS with the $p$-value of $<0.05$. Group B had better performance than group A. There was no significant difference found in MAIS as they had similar scores between the groups with the $p$-value of $>0.05$. Age group 3.1 to 4 years had similar results in all the tests which showed no significant difference with the $p$-value of $>0.05$. Both the groups performed equally in this age group. Overall auditory and speech-language performance were significantly better in group B than group A.

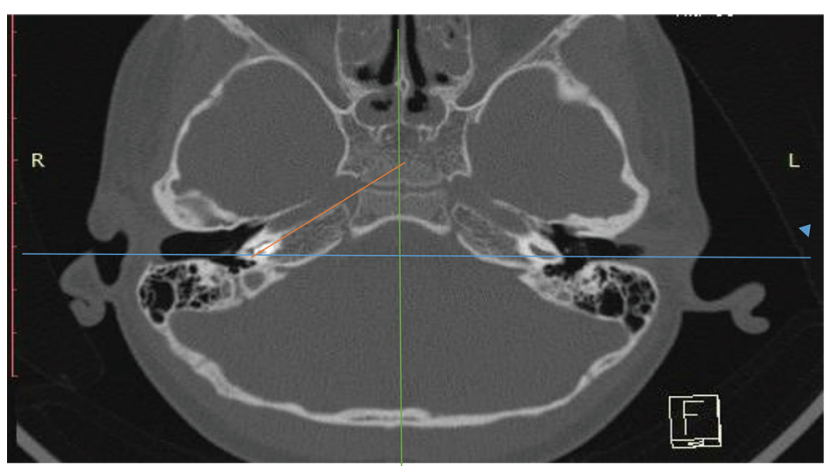

Fig. 5 Orange line indicates the electrodes in the basal turn of cochlea in relation to the sagittal plane. Straight line (blue) indicates the angle of the ear canal in relation to the sagittal plane which indicates that in transcanal approach alignment is not possible.

Table 3 Patients divided according to age

\begin{tabular}{|l|l|l|}
\hline Age range $(\mathbf{y})$ & Group A & Group B \\
\hline $0-1$ & 32 & 32 \\
\hline $1.1-2$ & 32 & 32 \\
\hline $2.1-3$ & 30 & 30 \\
\hline $3.1-4$ & 32 & 32 \\
\hline
\end{tabular}

\section{Discussion}

One of the first articles describing surgical complications related to cochlear implantation was published in 1991 by Cohen and Hoffman. ${ }^{3}$ The primary objective of this study is to compare the complications, pros and cons of both the approaches. End aural with inverted " $\mathrm{C}$ " skin incision used in the transcanal approach is a larger one compared with the post aural incision with snuggly fitting periosteal pocket used in posterior tympanotomy approach. End aural incision and mobilization of tympanomeatal flap in the transcanal approach has higher chances of tympanic membrane perforation, disturbed angulation of tympanic membrane, and postoperative granulation. ${ }^{9-11}$ The tunnel drilled in the attic region as described in the transcanal approach is a blind technique with a complex trough design. ${ }^{11}$ Even though it is assumed that the tunnel is away from the facial nerve, facial nerve injury is nevertheless possible, in cases of aberrant facial nerve, dehiscent facial nerve canal, and in cases of re-implantation. Damage to the facial nerve can be minimized in posterior tympanotomy approach by correctly identifying the landmarks, position of the bony facial canal and using facial nerve monitoring, and also studying the precise course of facial nerve by computed tomography scans of petrous temporal bone. The electrode is always under vision during insertion through posterior tympanotomy approach. Electrode handling is minimal in case of posterior tympanotomy approach, while in the transcanal approach, the electrode array passes through three bends from transcanal well into the tunnel at its entry, exit from the tunnel, and then through the cochleostomy; complete alignment to the basal turn of cochlea is not possible in all cases. ${ }^{12,13}$ Cholesteatoma or severe retraction of pars tensa is a complication which occurs due to injury to the canal wall or tympanic annulus and thin external meatal wall. Bone resorption can lead to migration of keratinocytes. A few cases have been reported in literature till date, with an incidence varying from $1.4 \%$ in children to $5.4 \%$ in adults, although cholesteatoma occurs in patients without intraoperative injuries. Transcanal approach increases the opportunity for extrusion and epithelial ingrowth. Mostafa et al in 2014, described 125 cases in Egypt who underwent $\mathrm{Cl}$ by the modified transcanal approach. Six patients suffered chorda tympani injury; two had tympanic membrane perforation, another two patients showed electrode exposure, one of which required revision with one patient having severe infection. ${ }^{14}$ Lavinsky et al in

Table 4 Mean scores (CAP, SIR, MAIS, MUSS) as measured in two groups

\begin{tabular}{|c|c|c|c|c|c|c|c|c|c|}
\hline \multicolumn{2}{|c|}{ Age group } & \multicolumn{4}{|c|}{ Group A } & \multicolumn{4}{|c|}{ Group B } \\
\hline & & CAP & SIR & MAIS & MUSS & CAP & SIR & MAIS & MUSS \\
\hline 1. & $0-1 y$ & 5.3 & 2.8 & 28.9 & 24.7 & 5.7 & 3.2 & 30.3 & 27.1 \\
\hline 2. & $1.1-2 y$ & 4.7 & 2.6 & 23.8 & 19.8 & 5.1 & 2.8 & 29.1 & 24.9 \\
\hline 3. & $2.1-3 y$ & 3.5 & 2.3 & 24.8 & 20.9 & 4.7 & 2.8 & 26.8 & 22.3 \\
\hline 4. & $3.1-4 y$ & 3.4 & 2 & 21.4 & 16.7 & 4 & 2.3 & 23.7 & 20.6 \\
\hline
\end{tabular}

Abbreviations: CAP, categories of auditory perception; MAIS, meaningful auditory integration scale; SIR, speech intelligibility rating. 
their study said that a combined approach (without drilling of tunnel) is a safe variation to $\mathrm{Cl}$ surgery. ${ }^{10}$ In this study, our results showed that the auditory performance, speech intelligibility, and use of language in day to day life were better in group B than group A as they had major and minor complications in the surgical approach. This could be one of the reasons for limited auditory and speech-language performance. Both the groups have shown increase in the scores as the time of implant use increased during the follow-up period.

Postoperative long-term speech performance gets affected according to the surgical strategy used. ${ }^{15}$ In our study, a few patients had poor auditory and speech performance among all the age groups. The reason could be surgical methods and strategies, and postoperative complications. In this study, we found that the children who were operated before 2 years of age had shown good improvement as compared with the age group between 2 and 4 years. Comparing the functional outcomes of cochlear implantation in children within 2 years of age and older children is limited. ${ }^{16}$ We analyzed the auditory and speech performance of seven children who had surgical revision in the group A. Their performances were poorer despite a functioning device. Auditory and speech performances remained the same or had little improvement after reimplantation. ${ }^{17}$

- Pros of transcanal approach include:

1. Better visualization of round window.

2. Avoidance of drilling of mastoid.

3. Thought of safety of facial nerve.

- Cons of transcanal approach include:

1. Entry of electrode array is at an angle with basal turn of cochlea.

2. Handling of electrodes as it stops in tunnel, needs to be pushed which may lead to kinking.

3. Complications are high due to involvement of ear canal and using it as conduit for electrodes.

In the Asian set up, a significant number of cases have been performed by this technique but only the advantages are explained by the physicians to the patients. Md El Anwar et al in 2016 cited after reviewing literature that non-mastoidectomy approach is valid in cases where conventional posterior tympanotomy is difficult to perform. ${ }^{9}$ Posterior tympanotomy approach has been practiced worldwide as highly successful surgical intervention with lesser rate of major complications; snuggly fitting subperiosteal pocket covers the receiver stimulator completely preventing displacement and implant exposure. And also, there is the advantage of modifying the surgical technique in patients with temporal bone abnormalities like low lying dura, antepost sigmoid, contracted mastoid, facial nerve anomalies, through the same incision.

\section{Conclusion}

Low complication rates when performed by experienced surgeon's make cochlear implantation with mastoidectomy and posterior tympanotomy approach a safe surgical procedure. We recommend that tunnel making in the transcanal approach should be avoided.

Surgeons performing posterior tympanotomy approach should be aware of these variations and should be able to modify the surgical technique. The transcanal approach is associated with increased chances of complications like injury to tympanic membrane, annulus, and damage to the posterior meatal wall, retraction of tympanic membrane, EAC granulations, and cholesteatoma. Although posterior tympanotomy also can damage tympanic membrane annulus, this can be minimized by surgical expertise to confine it to the level of a short process of incus. Both techniques have advantages and disadvantages in them and should be performed after careful consideration. One's innovative instincts with respect to each technique should be appropriately balanced and considered with care when treating this major disability.

\section{Ethical Approval}

The study was approved by the Institutional Ethics Committee.

\section{Data Availability}

Data are available for review on request from the authors due to privacy/institutional ethical restrictions.

\section{Conflict of Interest \\ None declared.}

\section{References}

1 Brito R, Monteiro TA, Leal AF, Tsuji RK, Pinna MH, Bento RF. Surgical complications in 550 consecutive cochlear implantation. Rev Bras Otorrinolaringol (Engl Ed) 2012;78(3): 80-85

2 Kirtane MV, Chavan KP. Cochlear implant surgical technique: our experience. Ann Otol Neurotol 2018;1(1):7-10

3 Cohen NL, Hoffman RA. Complications of cochlear implant surgery in adults and children. Ann Otol Rhinol Laryngol 1991;100(9 Pt 1) :708-711

4 Kiratzidis T, Arnold W, Iliades T. Veria operation updated. I. The transcanal wall cochlear implantation. ORL J Otorhinolaryngol Relat Spec 2002;64(6):406-412

5 Kronenberg J, Migirov L, Dagan T. Suprameatal approach: new surgical approach for cochlear implantation. J Laryngol Otol 2001;115(4):283-285

6 Häusler R. Cochlear implantation without mastoidectomy: the pericanal electrode insertion technique. Acta Otolaryngol 2002;122(7):715-719

7 Kronenberg J, Migirov L. The suprameatal approach: an alternative surgical technique for cochlear implantation. Cochlear Implants Int 2006;7(3):142-147

8 Postelmans JT, Grolman W, Tange RA, Stokroos RJ. Comparison of two approaches to the surgical management of cochlear implantation. Laryngoscope 2009;119(8):1571-1578

9 El-Anwar MW, ElAassar AS, Foad YA. Non-mastoidectomy cochlear implant approaches: a literature review. Int Arch Otorhinolaryngol 2016;20(2):180-184

10 Lavinsky-Wolff M, Lavinsky L, Dall'Igna C, Lavinsky J, Setogutti E, Viletti MC. Transcanal cochleostomy in cochlear implant surgery: long-term results of a cohort study. Rev Bras Otorrinolaringol (Engl Ed) 2012;78(2):118-123

11 SürmelioğluÖ,Özdemir S, TarkanÖ, et al. Alternative techniques in cochlear implantation. J Int Adv Otol 2016;12(1):109-112 
12 Tarabichi M,NazhatO, KassoumaJ, Najmi M.Endoscopiccochlear implantation: call for caution. Laryngoscope 2016;126(3): 689-692

13 Torres R, Drouillard M, De Seta D, et al. Cochlear implant insertion axis into the basal turn: a critical factor in electrode array translocation. Otol Neurotol 2018;39(2):168-176

14 Mostefa EB, Ezzat FW, Mogui MA. Approach for cochlear implantation: technique and results. Advances in Otolaryngology 2014;(2014):1-5

15 Jang JH, Park MH, Song JJ, et al. Long-term outcome of cochlear implant in patients with chronic otitis media: one-stage surgery is equivalent to two-stage surgery. J Korean Med Sci 2015;30(1):82-87

16 Hehar SS, Nikolopoulos TP, Gibbin KP, O’Donoghue GM. Surgery and functional outcomes in deaf children receiving cochlear implants before age 2 years. Arch Otolaryngol Head Neck Surg 2002;128(1):11-14

17 Kim CS, Kim DK, Suh MW, Oh SH, Chang SO. Clinical outcomes of cochlear reimplantation due to device failure. Clin Exp Otorhinolaryngol 2008;1(1):10-1 